\title{
A Genetic Algorithm for Constructing Broadcast Trees with Cost and Delay Constraints in Computer Networks
}

\author{
Ahmed Y. Hamed ${ }^{1}$ and Ghazi Al-Naymat ${ }^{2}$ \\ ${ }^{1}$ College of Applied Studies and Community Services, University of Dammam, KSA \\ ${ }^{2}$ Deanship of Information \& Communication Technology,University of Dammam, KSA
}

\begin{abstract}
We refer to the problem of constructing broadcast trees with cost and delay constraints in the networks as a delay-constrained minimum spanning tree problem in directed networks. Hence it is necessary determining a spanning tree of minimal cost to connect the source node to all nodes subject to delay constraints on broadcast routing. In this paper, we proposed a genetic algorithm for solving broadcast routing by finding the low-cost broadcast tree with minimum cost and delay constraints. In this research we present a genetic algorithm to find the broadcast routing tree of a given network in terms of its links. The algorithm uses the connection matrix of the given network to find the spanning trees and considers the weights of the links to obtain the minimum spanning tree. Our proposed algorithm is able to find a better solution, fast convergence speed and high reliability. The scalability and the performance of the algorithm with increasing number of network nodes are also encouraging.
\end{abstract}

\section{KEYWORDS}

Computer Networks, Broadcast Trees, Genetic Algorithm

\section{INTRODUCTION}

In computer networks, broadcasting refers to transmitting a packet that will be received by every device in the network [1]. In practice, the scope of the broadcast is limited to a broadcast domain. Broadcasting a message is in contrast to unicast addressing in which a host sends datagrams to another single host identified by a unique IP address.

Broadcast is a communication function that a node, called the source, sends messages to all the other nodes in the networks. Broadcast is an important function in applications of ad-hoc networks, such as in cooperative operations, group discussions, and so on. Broadcast routing is finding a broadcast tree, which is rooted from the source and contains all the nodes in the network. The cost of a broadcast is defined as the sum of cost of all the links that transmit the broadcast message in the broadcast tree.

The spanning tree is defined as a connected sub-graph of $G(N, E)$ that contains all its nodes and nocycles[2][3]. A simple approach is to use elementary tree-transformation which is based on the addition of a chord and deletion of an appropriate branch from a spanning tree. Thus, starting from any spanning tree of the graph with $\mathrm{n}$ nodes, one can generate all spanning trees by successive cyclic exchange[2]. This straightforward approach is difficult to computerize. So, 
Aggarwal[4] presented another algorithm, uses Cartesian products of (n-1) vertex cut sets whose elements are the branches connected to any of the (n-1) nodes of the graph.

In this paper, we present a genetic algorithm to find the broadcast routing tree of a given network in terms of its links. The algorithm uses the connection matrix of a given network to find the spanning trees, and then it considers the weights of the links to obtain the minimum spanning tree.

The paper is organized as follows: Section 2takes account of the related work. In Section 3, we define the problem that we are aiming to solve. Section 4 highlights some facts about the spanning tree structure. Section 5 discusses our proposed algorithm and all its steps. In Section 6 , we provide our experiments that demonstrate our technique's findings. Finally, we provide our conclusions in Section 7. The table below lists most of the used notations in this paper.

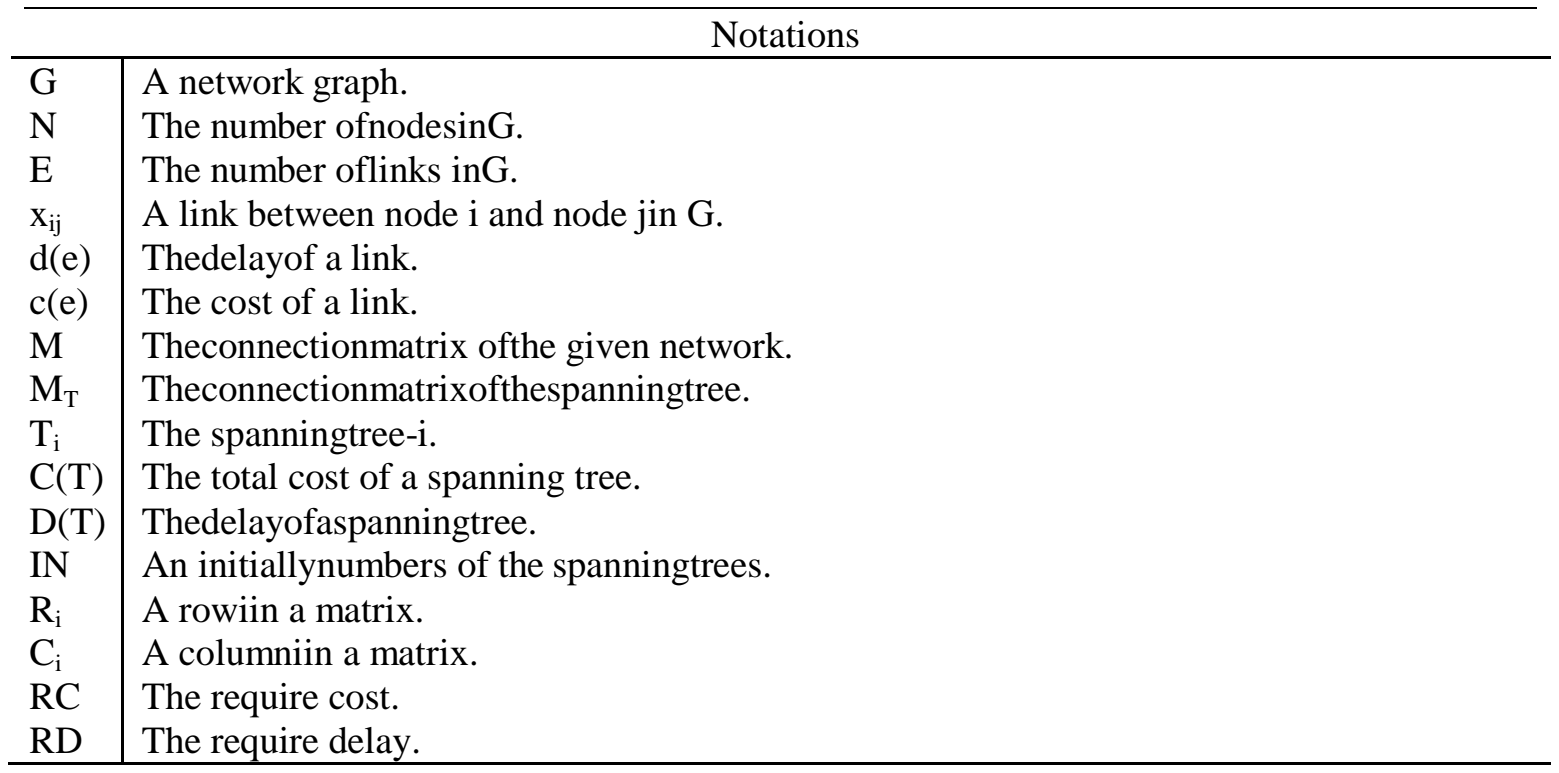

\section{RELATED WORK}

The minimum spanning tree problem (MSTP, Busacker[5] has been well studied in this framework, and efficient algorithms are widely known to solve this problem[6][7]. With minor changes, the same algorithms can be used to find the maximum spanning tree as well.

Younes[8] presented an algorithm to find the spanning trees of the computer network in terms of its links for using it in computing the network reliability.

Young[3]discussed these problems and described the first distributed algorithm for constructing minimal spanning trees. The algorithm and the principles and techniques underlying its design find application in large communication networks and large multiprocessor computer systems.

Chekuri [9] focused on scheduling to minimize two related objectives: the maximum response time and maximum delay factor. 
Fard [10] demonstrated how the spanning trees of a network can be utilized to determine a node pair across which a link should be added in order to achieve the maximum all-terminal reliability improvement in a network.

Yamada [11] formulated the spanning tree detection problem, and developed heuristic as well as exact algorithms to solve the problem. With these algorithms, problems with up to 1000 nodes have been solved within a few seconds. The algorithms have been extended to list up all the spanning trees of an arbitrary specified weight.

Kardan[12] presented the complete version of DBMST that regards packet loss and dynamism of the network topology and provides reliable communication. The proposed method decreases the power consumption of reliable broadcasting up to $15 \%$ in comparison with the basic version. In DBMST, network structure adapts to topology variations quickly while it takes several hours for other methods to find out variations in network.

Salama [13] formulated the problem of constructing broadcast trees for real-time traffic with delay constraints in networks with asymmetric link loads as a delay-constrained minimum spanning tree (DCMST) problem in directed networks.

The Delay-Constrained Capacitated Minimum Spanning Tree (DC-CMST) is a recently proposed problem which arises in the design of the topology of communications networks. The DC-CMST proposes the joint optimization of the network topology in terms of the traffic capacity and its mean time delay[14]. He presented an evolutionary algorithm which uses Dandelion-encoding is proposed to solve the problem.

Younes[15] presented the broadcast routing algorithms that aim to minimize the cost of the routing path.

Sameh [16]presented an algorithm to manipulate the problem of minimizing the mean completion delay in wireless broadcast for instantly decodable network coding.

Salama [17] formulated the problem of constructing broadcast trees for real-time traffic with delay constraints in networks with asymmetric link loads as a delay-constrained minimum spanning tree (DCMST) problem in directed networks. They proved that this problem is NPcomplete, and they proposed an efficient heuristic to solve the problem based on Prim's algorithm for the unconstrained minimum spanning tree problem. This is the first heuristic designed specifically for solving the DCMST problem.

\section{Problem description}

Given a network in which each link has a fixed cost and delay, find a broadcast tree such that the cost of the broadcast tree is minimized and delay is constrained. Thus, we present a genetic algorithm to find the broadcast routing tree of a given network in terms of its links which will solve our problem. The algorithm uses the connection matrix of a given network to find the spanning trees, and also is based on the weights of the links to obtain the minimum spanning tree. The proposed algorithm goal is able to find a better solution, fast convergence speed and high reliability when obtaining the spanning trees. Extensive simulations have been conducted and the results have demonstrated the efficiency of the proposed algorithms

The definition of the problems as follows: 
A network is usually represented as a weighted directed graph $G=(N, E)$, where $\mathrm{N}$ denotes the set of nodes and $\mathrm{E}$ denotes the set of communication links connecting the nodes. $|N|$ and $|E|$ denote the number of nodes and links in the network respectively.

Any link e $\in \mathrm{E}$ has a cost $c(e)$ and a delay $d(e)$ associated with it. $c(e)$ and $d(e)$ may take any positive real values.

A spanning tree $T(s) \ni E$ is rooted at a source node $s \in V$ and contains exactly one path from s to any node $v \in(V-\{s\})$. The total cost of a tree $T(s)$ is simply:

$$
\operatorname{Cost} T s=\underset{e \in T s}{c e}
$$

A path $P T s, v \in T s$ is the set of tree links connecting s to $v \in V$.The cost of the path $P(T S, v)$ is:

$$
\operatorname{cost} P T s, v=\underbrace{}_{e \in P(T s, v))} c(e)
$$

And the delay of that path is:

$$
\text { Delay } P T s, v=\underset{e \in P(T s, v))}{d e}
$$

Thus the maximum delay of a spanning tree is:

$$
\text { Max_Delay } T s=\max _{v \in V}(\text { Delay } P T S, v
$$

Our problem in directed networks is: constructs the spanning tree $\mathrm{T}(\mathrm{s})$ rooted at $\mathrm{s}$ that has minimum total cost among all possible spanning trees rooted at $\mathrm{s}$ which have a maximum delay less than or equal to a given delay constraint D.

The problem can be expressed as: Given a directed network $G=(V, E)$, a positive $\operatorname{cost} c(e)$ for each e $\in E$, a source $s \in V$, a positive delay constraint RD and a positive cost RC, find a spanning tree $\mathrm{T}(\mathrm{s})$ that satisfy:

$$
\text { Cost } T s \leq R C
$$

Where $\mathrm{RC}$ is the require cost.

$$
\text { Max_Delay } T s \leq R D
$$

Where RD id the require delay. 


\section{The Spanning Tree}

A tree $T$ is considered as a spanning tree of graph $G$ if $T$ is a connected sub graph of $G$ and contains all nodes of $\mathrm{G}$. A link in $\mathrm{T}$ is called a branch of $\mathrm{T}$, while a link of $\mathrm{G}$ that is not in $\mathrm{T}$ is called chord. For a connected graph of $\mathrm{N}$ nodes and $\mathrm{E}$ links, the spanning tree has $(\mathrm{N}-1)$ branches and $(\mathrm{E}-\mathrm{N}+1)$ chords.

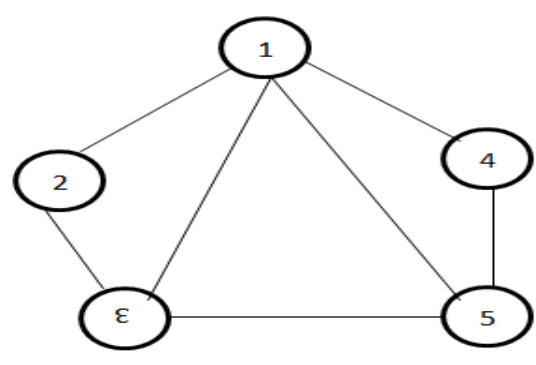

Graph G

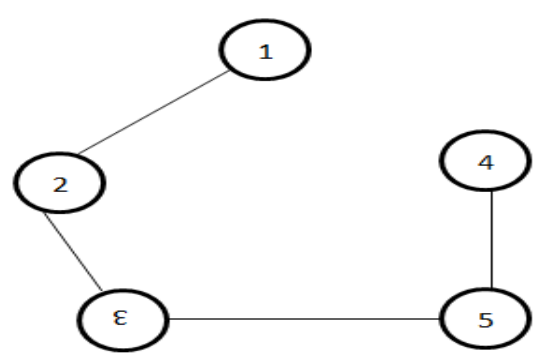

Spanning tree of $G$

I.e., the characterization of the spanning tree as:

- Contains(N-1)edges.

- ContainsallnodesofG.

- Isa connectedsubgraphofG.

The following subsections detail the above three characterizations of the spanning tree.

\subsection{TheSpanningTreeContainingallNodes}

In our proposed algorithm, one of the main steps is to validate that the spanning tree contains all nodes of the given network.To illustrate that, we consider a network of 8 nodes and 13 edges as shown in figure1.

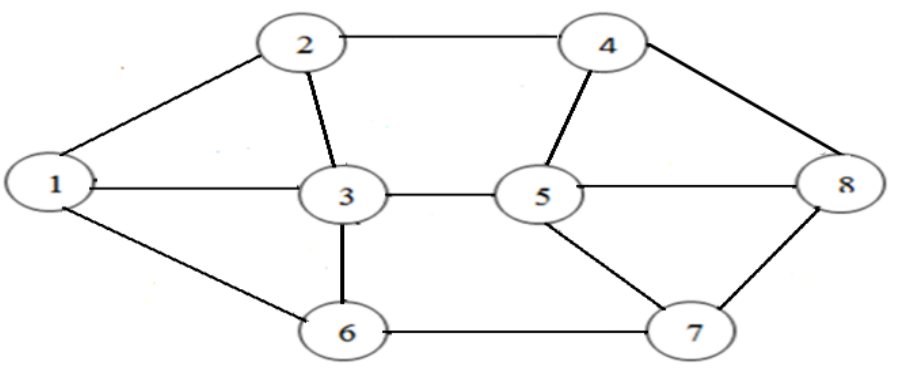

Figure 1asamplenetwork

The edges $1-2,1-3,1-6,2-4,3-5,6-7,7-8$ of the above figure represent a spanning tree $\mathrm{T}$ as shown in Figure 2. The tree $\mathrm{T}$ contains 7 edges (N-1 edges) this satisfies the first feature of the spanning tree (it contains $\mathrm{N}-1$ edges). The tree also contains all nodes of the above figure and this is the second feature. Finally,the tree forms a connected graph which means that all of its nodes are connected and this is the third feature of the spanning tree. 


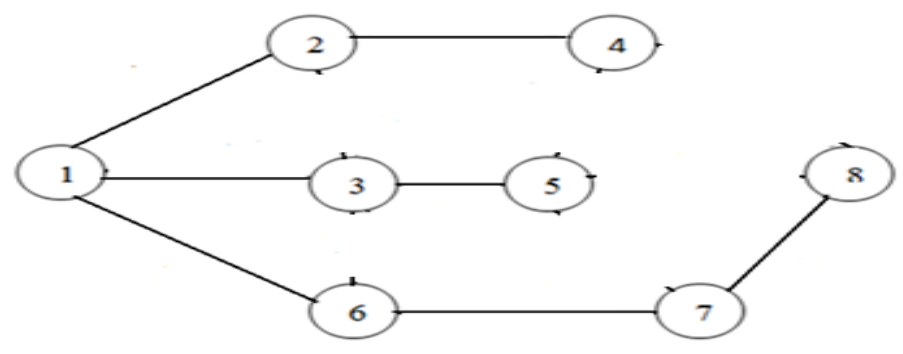

Figure 2A spanning tree of Figure 1.

The connection matrix $\mathrm{M}$ describes all direct connections between the nodes of a graph. The weighted connection matrix is a square matrix of dimension $\mathrm{n} \times \mathrm{n}$. Entries of $\mathrm{M}$ are defined such that the entry Mij at the intersection of row $\mathrm{i}$ and column $\mathrm{j}$, represents a connection from node $\mathrm{i}$ to node $\mathrm{j}$. The rules that define a weighted connection matrix are as follows:

$$
M_{i j}=\begin{aligned}
& 1 \text { if node } i \text { and node } j \text { are connected } \\
& 0 \text { otherwise }
\end{aligned}
$$

That is, the connection matrix of the Figure 1is:

$$
M=\begin{array}{llllllll}
0 & 1 & 1 & 0 & 0 & 1 & 0 & 0 \\
1 & 0 & 1 & 1 & 0 & 0 & 0 & 0 \\
1 & 1 & 0 & 0 & 1 & 1 & 0 & 0 \\
0 & 1 & 0 & 0 & 1 & 0 & 0 & 1 \\
0 & 0 & 1 & 1 & 0 & 0 & 1 & 1 \\
1 & 0 & 1 & 0 & 0 & 0 & 1 & 0 \\
0 & 0 & 0 & 0 & 1 & 1 & 0 & 1 \\
0 & 0 & 0 & 1 & 1 & 0 & 1 & 0
\end{array}
$$

Figure 3Theconnectionmatrixof tree inFigure 1

The spanning tree $\mathrm{T}$ contains all nodes of the given network, if all rows and columns of $\mathrm{M}_{\mathrm{T}}$ are non-zero elements $\left(\mathrm{M}_{\mathrm{T}}\right.$ is the connection matrix of $\mathrm{T}$, which is created using Equation (7)).

For example, if we consider the above spanning tree (Figure 2), the connection matrix $\mathrm{M}_{\mathrm{T}}$ is in the following form:

$$
M_{T}=\begin{array}{llllllll}
0 & 1 & 1 & 0 & 0 & 1 & 0 & 0 \\
1 & 0 & 0 & 1 & 0 & 0 & 0 & 0 \\
1 & 0 & 0 & 0 & 1 & 0 & 0 & 0 \\
0 & 1 & 0 & 0 & 0 & 0 & 0 & 0 \\
0 & 0 & 1 & 0 & 0 & 0 & 0 & 0 \\
1 & 0 & 0 & 0 & 0 & 0 & 1 & 0 \\
0 & 0 & 0 & 0 & 0 & 1 & 0 & 1 \\
0 & 0 & 0 & 0 & 0 & 0 & 1 & 0
\end{array}
$$

Figure 4Theconnectionmatrixofthe above spanningtree in Figure 2 
In Figure 4, we notice that the matrix does not contain any row or column that all its elements are zeros. Hence, the spanning tree of Figure 2 contains all the nodes of the given network (Figure 1).

\subsection{The Spanning Tree is a Connected Sub Graph.}

In the proposed algorithm, we prove that the spanning tree is a connected sub graph as follows: A graph $G$ is connected if there is at least one path between every pair of nodes $i$ and $j$, which minimally requires a spanning tree with $(N-1)$ edges.

The sub graph is connected if all its nodes are connected. To confirm if a spanning tree $\mathrm{T}$ is a connected sub graph or not, we can repeat the union between the elements of both $\mathrm{R} 1$ and $\mathrm{Rj}$ in the matrix $\mathrm{M}_{\mathrm{T}}$ where $\mathrm{Rj}$ is a list of all indexes of the none zero elements in $\mathrm{R} 1$ and it. On other hand, we can use the following relation (8) which was used by Younes[8]:

Do

\{

$$
R_{1}=R_{1} R_{j \in R_{1}} 8
$$

\} while jis all indexes of the non zeroelementsin R1.

$$
M_{T}=\left[\begin{array}{llllll}
0 & 1 & 0 & 0 & 0 & 0 \\
1 & 0 & 1 & 0 & 0 & 0 \\
0 & 1 & 0 & 0 & 1 & 0 \\
0 & 0 & 0 & 0 & 1 & 1 \\
0 & 0 & 1 & 1 & 0 & 0 \\
0 & 0 & 0 & 1 & 0 & 0
\end{array}\right]
$$

Figure 5Example to check the sub routine in Equation (8)

We use the example in Figure 5 to illustrate our method in checking the connectivity of the sub graph. Below you see the steps the union operations are performed between R1 and $\mathrm{Rj}$ and how $\mathrm{R} 1$ is updated in each iteration.

$$
\begin{array}{llllllllllllllllllll}
\mathrm{R} 1=\mathrm{R}_{1} \cup \mathrm{R}_{2}: & 0 & \underline{1} & 0 & 0 & 0 & 0 & \cup & 1 & 0 & 1 & 0 & 0 & 0=1 & 1 & 1 & 0 & 0 & 0 \\
\mathrm{R} 1=\mathrm{R}_{1} \cup \mathrm{R}_{3}: & 1 & 1 & 1 & 0 & 0 & 0 & \cup & 0 & 1 & 0 & 0 & 1 & 0=1 & 1 & 1 & 0 & 1 & 0 \\
\mathrm{R} 1=\mathrm{R}_{1} \cup \mathrm{R}_{5}: & 1 & 1 & 1 & 0 & 1 & 0 & \cup & 0 & 0 & 1 & 1 & 0 & 0=1 & 1 & 1 & 1 & 1 & 0 \\
\mathrm{R} 1=\mathrm{R}_{1} \cup \mathrm{R}_{4}: & 1 & 1 & 1 & 1 & 1 & 0 & \cup & 0 & 0 & 0 & 0 & 1 & 1=1 & 1 & 1 & 1 & 1 & 1 & 1 \\
\mathrm{R} 1=\mathrm{R}_{1} \cup \mathrm{R}_{6}: & 1 & 1 & 1 & 1 & 1 & 1 & \cup & 0 & 0 & 0 & 1 & 0 & 0=1 & 1 & 1 & 1 & 1 & 1
\end{array}
$$


If there exists one zero element in $\mathrm{R} 1$, this mean the spanning tree $\mathrm{Ti}$ is not aconnected sub graph.

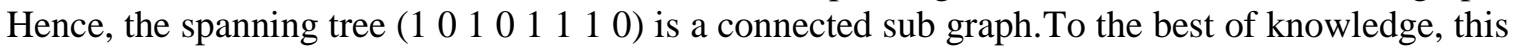
technique is a unique one and not previously used to validate whether the tree is connected or not.

\section{The Proposed Genetic Algorithm (GA)}

In the proposed GA, each spanning tree is represented by a binary string that can be used as a chromosome. Each element of the chromosome represents a link in the network topology. So, for a network of $\mathrm{N}$ nodes and $\mathrm{E}$ edges, there are $\mathrm{E}$ string components in each candidate solution $\mathrm{x}$. Each chromosome must contains N-1 none zero elements. For example, the spanning tree of Figure 2is represented as a chromosome as shown in Figure 6.

\begin{tabular}{|c|c|c|c|c|c|c|c|c|c|c|c|c|}
\multicolumn{1}{c}{$1-2$} & $1-3$ & $1-6$ & \multicolumn{1}{c}{$2-3$} & \multicolumn{1}{c}{$2-4$} & $3-5$ & $3-6$ & $4-5$ & $4-8$ & $5-7$ & $5-8$ & $6-7$ & $7-8$ \\
\hline 1 & 1 & 1 & 0 & 1 & 1 & 0 & 0 & 0 & 0 & 0 & 1 & 1 \\
\hline
\end{tabular}

Figure 6Thechromosome form

\subsection{Initial Population}

The initial population is generated according to the following steps:

1. Achromosomex intheinitialpopulationcanbe generatedas shownin Figure 6.

2. ThechromosomemustcontainonlyN-1nonezeroelements

3. Repeatsteps1 to2 to generatepop_size(population size), numberofchromosomes.

\subsection{Fitness Function}

The fitness function is used in each iteration of the genetic algorithm to evaluate the quality of all proposed solutions to the problem in the current population. Hence; the fitness function is used as a filter function to eliminate all non-candidate solutions. The fitness function used to verify the solution is as follow:

- The chromosome must contain all nodes of the network.

- $\quad$ The chromosome must be a connected sub graph using Equation (8).

\subsection{Genetic Crossover Operation}

The crossover operation is used to breed a child from two parents by one cut point. The crossover operation will perform if the crossover ratio $(\mathrm{Pc}=0.90)$ is verified. The cut point israndomly selected. The crossover operation is performed as follows:

- Randomly selecttwochromosomesfromthecurrentpopulation.

- Randomlyselectthecutpoint

- Fillthecomponentsofthechromosome

a. Bytakingthecomponentsofthefirstchromosome(fromthefirst genetothecut point)andfill uptothechild.

b. Also, tacking the components of the second chromosome (from the cut point+1 to the last gene) and fill up to the child. 
The offspring generated by crossover operation is shown in Figure 7.

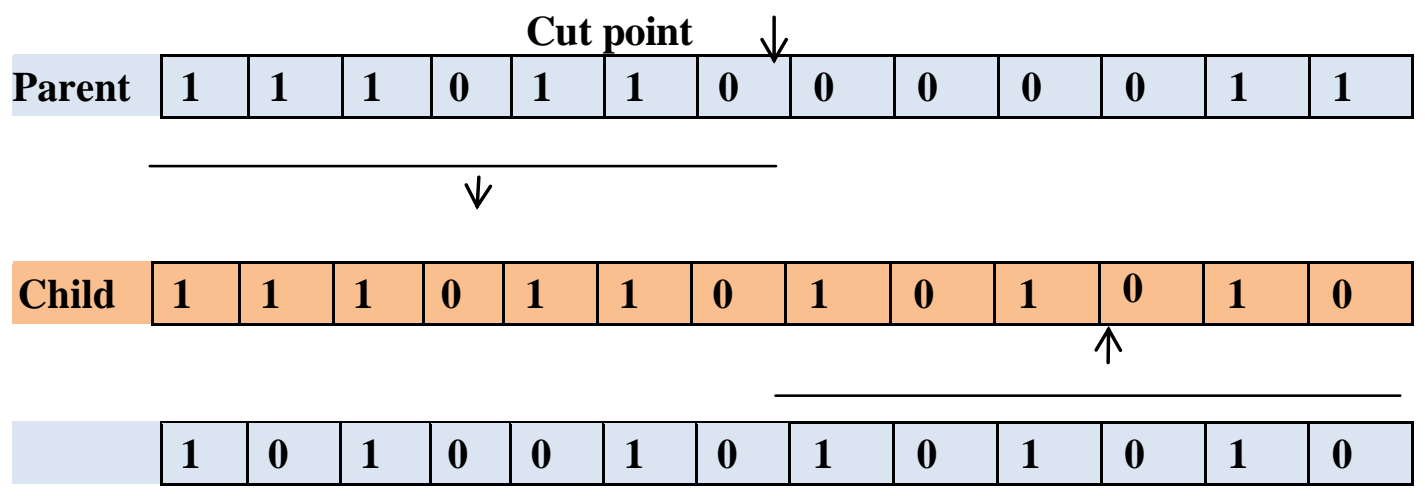

Figure 7 Example of the crossover operation

\subsection{Genetic Mutation Operation}

The mutation operation is performed on bit-by-bit basis. In the proposed approach, the mutation operation will be performed if the mutation ratio $(\mathrm{Pm})$ is verified. The $\mathrm{Pm}$ in this approach is chosen experimentally to be 0.02 . The point to be mutated is selected randomly. The offspring generated by mutation is shown in Figure 8 .

\begin{tabular}{|l|l|l|l|l|l|l|l|l|l|l|l|l|}
\hline 1 & 1 & 1 & 0 & 1 & 1 & 0 & 1 & 0 & 0 & 0 & 1 & 0 \\
\hline 1
\end{tabular}
\begin{tabular}{|l|l|l|l|l|l|l|l|l|l|l|l|l|}
\hline 1 & 1 & 1 & 0 & 1 & 1 & 1 & 1 & 0 & 0 & 0 & 1 & 0 \\
\hline
\end{tabular}

Figure 8 Example of the mutation operation.

\subsection{The Algorithm}

The following pseudocodeillustrates the use of our assumptions and proposed functions to find the minimum spanning tree.

Algorithm Find Minimum Spanning Tree

Input : Set the parameters: pop_size, max_gen, RC,RD, $\mathrm{P}_{\mathrm{m}}, \mathrm{P}_{\mathrm{c}}$

Output : Minimum Spanning Tree

1. Generatetheinitialpopulationaccording to the steps in Section5.1.

2. gen $\leftarrow 1$.

3. While $($ gen $<=$ max_gen $)$ do \{

4. $\mathbf{P} \leftarrow 1$

5. While $\left(P<=p o p \_s i z e\right)$ do \{

6. Geneticoperations
a. Obtainchromosomesofthe newpopulation.
b. Selecttwochromosomesfromthepopulationrandomly,
c. Applycrossoveraccordingto $\mathrm{P}_{\mathrm{c}}$ parameter $\left(\mathrm{P}_{\mathrm{c}}>=0.90\right)$, 
d. Mutatethe newchildaccordingto $P_{m}$ parameter $\left(\mathrm{P}_{\mathrm{m}}<=0.02\right)$,

e. Compute the fitness function according to section 5.2

f. If the result of fitness function is false then discard the child and go to step 6.b.

7. Computethe totalcostofthenewchild $\mathrm{C}(\mathrm{x})$ accordingtoEquation (1).

8. Find all the paths from the source node to all the nodes in the child ( $\mathrm{P} T \mathrm{~T}, \mathrm{v})$.

9. Compute the delay of each path in the child (Delay $\mathrm{P} T \mathrm{~S}, \mathrm{~V}$ ) according to Equation (3).

10. Compute the maximum delay of the child (Max_Dealy T s ) according to Equation (4).

11. If the $\mathrm{C}(\mathrm{x})<=\mathrm{RC}$ and Max_Dealy $\mathrm{T} \mathrm{s} \leq \mathrm{RD}$ then stop

12. Savethischildasa candidatesolution.

13. $\mathrm{P} \leftarrow \mathrm{P}+1$.

14. $\}$

15. Setgen $=$ gen +1

16. if gen >max_gen then stop

17. $\}$

\section{EXPERIMENTAL RESULTS}

The proposed algorithm is implemented using Borland $\mathrm{C}++$ Ver. 5.5 and the initial values of the parameters are: population size (pop-size $=20)$, maximum generation $(\max -\mathrm{gen}=250), \mathrm{Pc}=0.90$, $\mathrm{Pm}=0.02$. We have applied our proposed technique more than 20 cases from 4 nodes until 20 nodes. The technique reads the connection matrix of the given network and the cost and delay of each link of that network. Then it generates the connection matrix, cost and delay of each link of other networks. Two scenarios are used to test and validate the proposed technique. The subsections below demonstrate that.

\subsection{Scenario One}

The algorithmwas applied on two cases of known data (read the connection matrix of the given network, the cost and delay of each link). The first case is a network with 8 nodes and 13 links and the second case is a network with 16 nodes and 28 links.

\subsubsection{Case 1:}

We consider the given network as shown in Figure 1 ( 8 nodes and 13 links), the connection matrix as shown in Figure 2, and the cost and delay for each link are shown in Table 1.

Table 1 the cost and delay of the links

\begin{tabular}{|c|c|c|}
\hline Link & Cost & Delay \\
\hline $1-2$ & 16 & 1 \\
\hline $1-3$ & 12 & 2 \\
\hline $1-6$ & 28 & 3 \\
\hline $2-3$ & 15 & 3 \\
\hline $2-4$ & 22 & 1 \\
\hline $3-5$ & 10 & 2 \\
\hline
\end{tabular}


International Journal of Computer Networks \& Communications (IJCNC) Vol.7, No.1, January 2015

\begin{tabular}{|l|l|l|}
\hline $3-6$ & 17 & 1 \\
\hline $4-5$ & 14 & 2 \\
\hline $4-8$ & 24 & 3 \\
\hline $5-7$ & 20 & 2 \\
\hline $5-8$ & 23 & 1 \\
\hline $6-7$ & 18 & 3 \\
\hline $7-8$ & 21 & 2 \\
\hline
\end{tabular}

The required cost and delay of the minimum spanning tree are: $\quad \mathrm{RC}<=110 \quad$ and $\quad \mathrm{RD}<=7$.

1) Theminimumspanningtree of the first caseis shown in Figure 9.

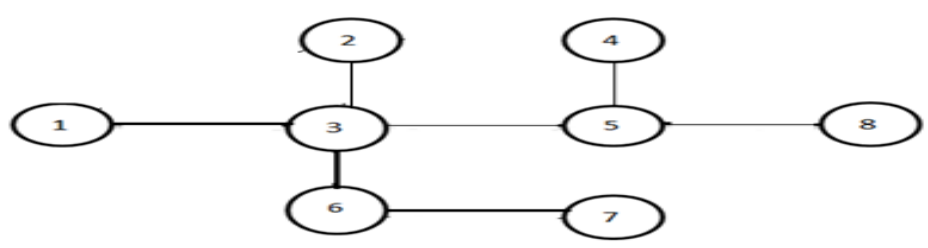

Figure 9 The minimum spanning tree of the network with 8 nodes and 13 links.

2) All paths of the minimum spanning tree $P(T(s), v)$ are shown in the Table 2.

Table 2 All the paths of the minimum spanning tree

\begin{tabular}{|c|cccc|c|c|}
\hline No. & \multicolumn{2}{|l|}{$\boldsymbol{P}(\boldsymbol{T}(\boldsymbol{s}), \boldsymbol{v})$} & cost $\boldsymbol{P} \boldsymbol{T} \boldsymbol{s}, \boldsymbol{v}$ & Delay $\boldsymbol{P} \boldsymbol{T}, \boldsymbol{v}$ \\
\hline 1 & 1 & 3 & 2 & 27 & 5 \\
\hline 2 & 1 & 3 & & & 12 & 2 \\
\hline 3 & 1 & 3 & 5 & 4 & 36 & 6 \\
\hline 4 & 1 & 3 & 5 & 22 & 4 \\
\hline 5 & 1 & 3 & 6 & 29 & 3 \\
\hline 6 & 1 & 3 & 6 & 7 & 47 & 6 \\
\hline 7 & 1 & 3 & 5 & 8 & 45 & 5 \\
\hline
\end{tabular}

3) The cost and maximum delay of the minimum spanning tree are: Cost $T S=109$ and Max_Delay $T s=6$.

4) The path of maximum delay is shown in Figure 10. 


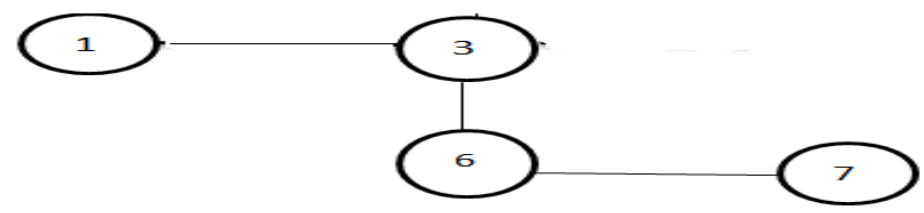

Figure 10 The path of maximum delay

\subsection{2. $\quad$ Case 2}

In this case, the algorithmwas applied on a network with 16 nodes and 28 links as shown in Figure 11. The cost and delay for each link are shown in the Table 3.

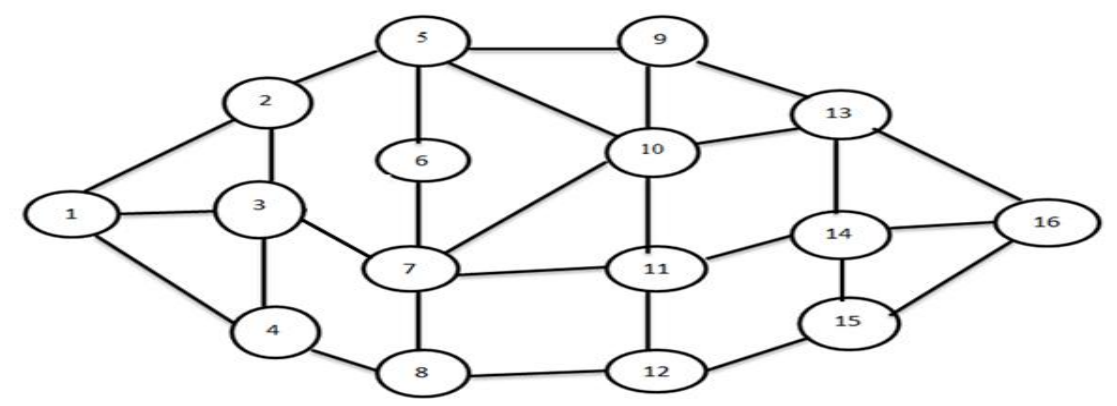

Figure 111 A network with 16 nodes and 28 links

Table 3 the cost and delay of the links

\begin{tabular}{|l|l|l|l|l|l|l|l|}
\hline$\#$ & \multicolumn{1}{|c|}{ Link } & Cost & Delay & $\#$ & Link & Cost & Delay \\
\hline 1 & $1-2$ & 10 & 1 & 15 & $7-11$ & 24 & 3 \\
\hline 2 & $1-3$ & 12 & 2 & 16 & $8-12$ & 25 & 2 \\
\hline 3 & $1-4$ & 15 & 3 & 17 & $9-10$ & 21 & 3 \\
\hline 4 & $2-3$ & 13 & 3 & 18 & $9-13$ & 10 & 2 \\
\hline 5 & $2-5$ & 14 & 2 & 19 & $10-11$ & 9 & 1 \\
\hline 6 & $3-4$ & 18 & 1 & 20 & $10-13$ & 18 & 2 \\
\hline 7 & $3-7$ & 23 & 2 & 21 & $11-12$ & 17 & 3 \\
\hline 8 & $4-8$ & 16 & 2 & 22 & $11-14$ & 16 & 1 \\
\hline 9 & $5-6$ & 19 & 2 & 23 & $12-15$ & 22 & 1 \\
\hline 10 & $5-9$ & 21 & 1 & 24 & $13-14$ & 11 & 1 \\
\hline 11 & $5-10$ & 13 & 3 & 25 & $13-16$ & 12 & 3 \\
\hline 12 & $6-7$ & 19 & 2 & 26 & $14-15$ & 13 & 2 \\
\hline 13 & $7-8$ & 18 & 1 & 27 & $14-16$ & 22 & 1 \\
\hline 14 & $7-10$ & 20 & 4 & 28 & $15-16$ & 18 & 2 \\
\hline
\end{tabular}

The required cost and delay of the case 2 are: $(\mathrm{RC}<=220 \quad$ and $\quad \mathrm{RD}<=15)$.

1) The minimum spanning tree is shown in Figure 12. 
International Journal of Computer Networks \& Communications (IJCNC) Vol.7, No.1, January 2015

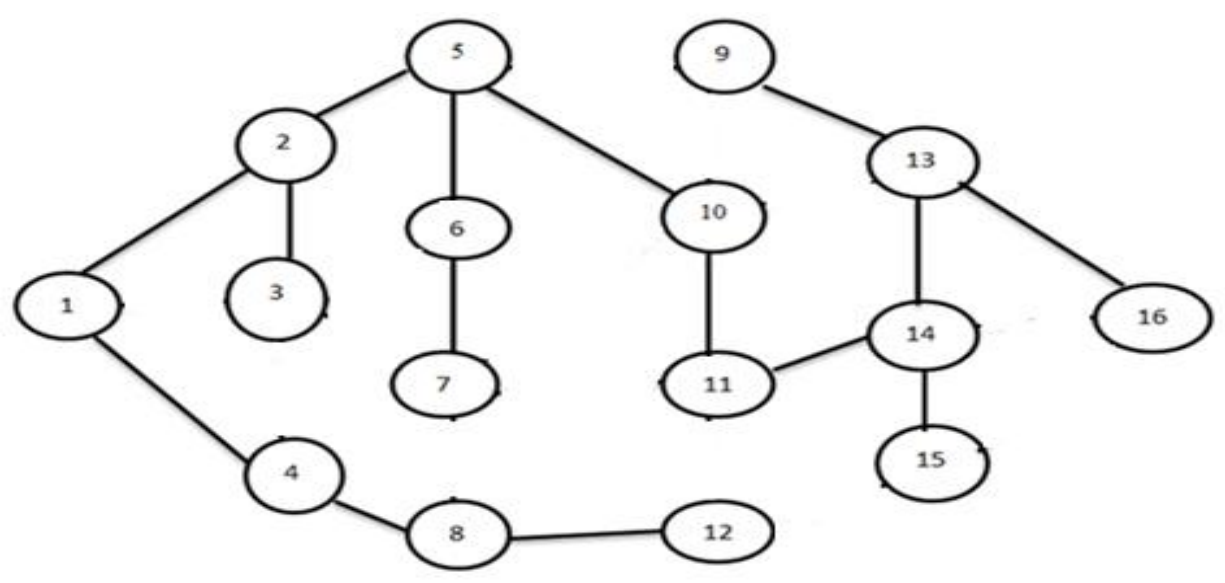

Figure 12 The minimum spanning tree of the Figure 11

2) All the paths of the minimum spanning tree are sown in the Table 4

Table 4 All the paths of the minimum spanning tree

\begin{tabular}{|c|c|c|c|c|c|c|c|c|c|c|}
\hline No. & \multicolumn{8}{|c|}{$P(T(s), v)$} & $\operatorname{cost} P T s, v$ & Delay $P T s, v$ \\
\hline 1 & 1 & 2 & & & & & & & 10 & 1 \\
\hline 2 & 1 & 2 & 3 & & & & & & 23 & 4 \\
\hline 3 & 1 & 4 & & & & & & & 15 & 3 \\
\hline 4 & 1 & 2 & 5 & & & & & & 24 & 3 \\
\hline 5 & 1 & 2 & 5 & 6 & & & & & 43 & 5 \\
\hline 6 & 1 & 2 & 5 & 6 & 7 & & & & 62 & 7 \\
\hline 7 & 1 & 4 & 8 & & & & & & 31 & 11 \\
\hline 8 & 1 & 2 & 5 & 10 & 11 & 14 & 13 & 9 & 85 & 6 \\
\hline 9 & 1 & 2 & 5 & 10 & & & & & 37 & 6 \\
\hline 10 & 1 & 2 & 5 & 10 & 11 & & & & 48 & 7 \\
\hline 11 & 1 & 4 & 8 & 12 & & & & & 56 & 7 \\
\hline 12 & 1 & 2 & 5 & 10 & 11 & 14 & 13 & & 75 & 9 \\
\hline 13 & 1 & 2 & 5 & 10 & 11 & 14 & & & 45 & 6 \\
\hline 14 & 1 & 2 & 5 & 10 & 11 & 14 & 15 & & 58 & 8 \\
\hline 15 & 1 & 2 & 5 & 10 & 11 & 14 & 13 & 16 & 87 & 12 \\
\hline
\end{tabular}

1) The cost and maximum delay of the minimum spanning tree are:

Cost $T s=215$ and Max_Delay $T s=12$.

2) The path of the maximum delay is shown in Figure 13 


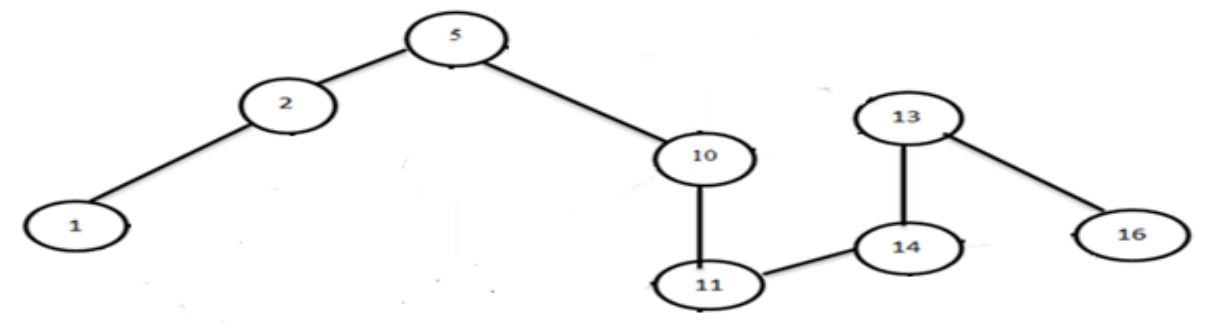

Figure 13 The path of the maximum delay

\subsection{Scenario Two}

The system is applied on 6 other cases (networks of size 5, 7, 10, 12, 14, and 20 nodes). In these cases the system generated the connection matrix, the cost and the delay for each link of the generated cases. The following table shows the results of these cases:

Table 5 The results of the generated cases in Scenario Two

\begin{tabular}{|c|c|c|c|c|}
\hline No. & Nodes & Links & Cost (T(s)) & Max-Delay (T(s)) \\
\hline 1 & 5 & 27 & 19 & 8 \\
\hline 2 & 7 & 12 & 42 & 11 \\
\hline 3 & 10 & 36 & 57 & 14 \\
\hline 4 & 12 & 22 & 62 & 15 \\
\hline 5 & 14 & 29 & 84 & 12 \\
\hline 6 & 20 & 47 & 137 & 19 \\
\hline
\end{tabular}

\section{Conclusions}

In this paper we presented a Genetic Algorithm (GA) to find the broadcast routing tree of a given network in terms of its links such that the cost of the broadcast tree is minimized and the delay is constrained. The algorithm used the connection matrix of a given network to find the spanning trees, and also is based on the weight of the links to obtain the minimum broadcast routing tree. The proposed GA is very useful and can be used in the large networks. The completeness and correctness of the proposed algorithm have been tested. This has proven that our proposed technique enabled us to obtain results faster, leading to save time and effort. In other words, the use of GA has played a major role in reducing the search space generated by the problem. In summary, our experimental results indicate that the algorithm is more efficiently than other heuristics. To the best of our knowledge, the structure and design of our method is designed for the first time to report a broadcast tree from a given network. This has made it very hard to find common features with other previous methods for comparison reasons.

\section{ACKNOWLEDGEMENTS}

The authors would like to thank the anonymous IJCNC reviewers for their insightful comments.This Project was funded by the Deanship of Scientific Research at the University of Dammam, KSA. 


\section{REFERENCES}

[1] A. Tanenboum and D. Wetherall, Computer Networks, Cloth: Prentice Hall, 2010.

[2] N. Deo, Graph theory with application to engineering and computer science, Englewood Cliffs, NJ: Prentice- Hall, 1974.

[3] H. Young, "A method for computing complex system reliability," IEEE Transaction on Reliability, vol. 21, no. 4, pp. 215-219, 1972.

[4] K. Aggarwal, "Reliability evaluation in computer communication networks," IEEE Transaction on Reliability, vol. 30, no. 1, pp. 32-35, 1981.

[5] R. Busacker, Finite graphs and networks: an introduction with applications, New York: McGraw-Hill, 1965.

[6] T. L. M. J. B. O. Ravindra K. Ahuja, Network Flows: Theory, Algorithms, and Applications, 1993.

[7] J. Joseph B. Kruskal, "On the shortest spanning subtree of a graph and the travelingsalesman problem," in Proceedings of the American Mathematical Society, 1956.

[8] A. Younes, "The spanning trees and computer networks reliability," Egyptian informatics journa, vol. 7, no. 2, 2006.

[9] C. Chekuri, " Minimizing Maximum Response Time and Delay Factor in Broadcast Scheduling," Lecture Notes in Computer Science, vol. 5757, pp. 444-455, 2009.

[10] N. Fard, "Spanning tree in all terminal network reliability expansion," Computer Communications, vol. 24, pp. 1348-1353, 2001.

[11] T. Yamada, "Heuristic and exact algorithms for the spanning tree detection problem," Computer \& Operation Research, vol. 32, pp. 239-255, 2005.

[12] A. Kardan and M. Kajbaf, "Distributed Broadcast Minimum Spanning Tree," in The 3rd International Conference on Intelligent Sensors, Sensor Networks and Information, Melbourne, Qld, 2007.

[13] H. Salama, D. Reeves and Y. Viniotis, "The delay-constrained minimum spanning tree problem," in Proceedings of the Second IEEE Symposium on Computers and Communications, Alexandria, 1997.

[14] M. Angel, "Solving the Delay-Constrained Capacitated Minimum Spanning Tree Problem Using a Dandelion-Encoded Evolutionary Algorithm," Simulated Evolution and Learning, vol. 5361, pp. 151$160,2008$.

[15] A. Younes, "Minimizing the broadcast routing in the computer networks," International Journal of Computer Engineering Science, vol. 2, no. 3, pp. 62-68, 2012.

[16] S. Sorour, E. S. R. Sr. and S. Valaee, "On Minimizing Broadcast Completion Delay for Instantly Decodable Network Coding," in IEEE International Conference on Communications, Cape Town, 2010.

[17] H. F. D. S. R. Salama and Y. Viniotis, “An Efficient Delay-Constrained Minimum Spanning Tree Heuristic," in Proceedings of the 5th International Conference on Computer Communications and Networks, 1996.

[18] K. Y. Dalal, “A Distributed Algorithm for Constructing Minimal Spanning Trees,” IEEE Transaction on Software Engineering, vol. 13, no. 3, pp. 398 - 405, 1987. 


\section{Authors}

Ahmed Younes Hamedreceived his PhD degree in Sept.1996 from South Valley University, Egypt. His research interests include Artificial Intelligence and genetic algorithms; specifically in the area of computer networks. Recently, he has started conducting a research in the area of Image Processing. Currently, he works as an Associate Professor in University of Dammam, KSA. Younes always publishes the outcome of his research in international journals and conferences.

Ghazi Al-Naymat received his PhD degree in May 2009 from the school of Information Technologies at The University of Sydney, Australia. His research interests include data mining and knowledge discovery; specifically in the area of spatial, spatio-temporal and time series applications. Recently, he has started conducting a research in the area of Cloud Computing and Big data analytics. Currently, he works as an Assistant Professor in University of Dammam, KSA. Al-Naymat always publishes the outcome of his research in international journals and conferences. 